\title{
Applicable Predictive Maintenance Diagnosis Methods in Service-Life Prediction of District Heating Pipes
}

\author{
Pakdad POURBOZORGI LANGROUDI ${ }^{1 *}$, Ingo WEIDLICH ${ }^{2}$ \\ ${ }^{1,2}$ HafenCity University Hamburg, Überseeallee 16, 20457 Hamburg, Germany
}

\begin{abstract}
Maintaining the supply chain in every industry is an important concern for the operators. The negative impacts of inappropriate maintenance could be discussed from different perspectives as well as capital loss, reputation loss, hazard and risk for lives, etc. In recent years, District heating $(\mathrm{DH})$ in the countries that employing this technology broadly, turned to a vital energy infrastructure for delivering heat from suppliers to the consumers. Therefore, the reliability of the system is of high importance for the public interest. The transition from reactive maintenance to proactive maintenance have improved a lot the reliability to the system. Currently, many industries are exploiting different forms of artificial intelligence (AI) to predict the failures and plan for interventions to increase the system efficiency. In this paper the different methods of predictive maintenance have been reviewed and the compatibility to apply on a DH network has been discussed.
\end{abstract}

Keywords - Artificial neural networks; asset management; condition-based maintenance; machine learning; proactive maintenance; system reliability

\begin{tabular}{|lll|}
\hline Nomenclature & & - \\
AHP & Analytic Hierarchy Process & - \\
AI & Artificial Intelligence & - \\
ANN & Artificial Neural Networks & - \\
CBM & Condition-based Maintenance & - \\
CNN & Convolutional Neural Networks & - \\
DH & District Heating & - \\
GMM & Gaussian Mixture Model & - \\
HMM & Hidden Markov Model & - \\
IoT & Internet of Things & - \\
LSTM & Long Short-term Memory & - \\
ML & Machine Learning & - \\
MLP & Multilayer Perceptron & - \\
PdM & Predictive Maintenance & - \\
PE & Polyethylene & \\
\hline
\end{tabular}

* Corresponding author.

E-mail address: pakdad.langroudi@hcu-hamburg.de 


\begin{tabular}{|lll|}
\hline PUR & Rigid Polyurethane & - \\
PvM & Preventive Maintenance & - \\
R2F & Run-to-Failure & - \\
RCM & Reliability-centred Maintenance & - \\
RNN & Recurrent Neural Networks & - \\
\hline
\end{tabular}

\section{INTRODUCTION}

All manufacturing or production plants in industries try to stabilize the production line and idealistically without any down time. This is not possible due to service-life limitation of the system components and at one moment the system stops and certain repairs are required to keep it running again. The actions to be performed to keep a system running is known as maintenance. The impacts of inappropriate maintenance in some industries sometimes could be unrecoverable as it dealt with safety issue. Maintenance is not only about safety, but also about asset management. Industry owners could bring efficiency to their system with proper maintenance strategy in order to minimize the cost and increase the reliability.

The maintenance cost itself could vary from application to application between $15 \%$ and $70 \%$ [1], [2]. These percentages are considerable, as if it was not necessary. Therefore, a certain share of profit could be considered as waste. According to [2], the maintenance effectiveness is about two-third cents of a dollar in U.S. This means 33 cents are wasted as a result of unnecessary or inappropriate maintenance strategies [2]. Thus, understanding different maintenance strategies, not only from the financial point of view but also from the environmental aspects are at importance to minimize the material consumption and have a reliable system as well.

Susto et al in [3] categorized the maintenance strategies into three main groups as follows:

1. Run-to-Failure (R2F);

2. Preventive Maintenance (PvM);

3. Predictive Maintenance (PdM).

$\mathrm{R} 2 \mathrm{~F}$ and PvM are the most common strategies in many sectors and well explained in [2], [4]-[8]. Recent evolution of digital world (e.g., Artificial Intelligence (AI), Internet of Things (IoT), Artificial Neural Networks (ANN), sensing \& inspection technologies, etc.), gives an unprecedented chance to improve upon existing maintenance operations and approach toward predictive maintenance. Therefore, as latest maintenance strategy, many sectors have employed PdM for their business. This trend has mainly observed in fields where reliability is at paramount importance, such as power plants, oil and gas industry, and utilities [9], [10].

In summary, Yongyi Ran et al. [6] listed the benefits, challenges, applicable and inappropriate applications for R2F, PvM, and PdM as in Table 1.

Table 1. Benefits, Challenges and Applications of R2F, PVM, And PdM [6]

\begin{tabular}{lllll}
\hline & Benefits & Challenges & Applicable where & Inappropriate where \\
\hline R2F & Maximum utilization and & Unplanned downtime & Redundant, or non- & $\begin{array}{l}\text { Equipment failure } \\
\text { croduction value }\end{array}$ \\
cower prevention cost & High spare parts & inventory cost & Repairing equipment & 24/7 equipment \\
& & Potential further damage & with low cost after & availability is \\
& for the equipment & breakdown & necessary \\
& Higher repair cost & & \\
& & &
\end{tabular}




\begin{tabular}{lllll}
\hline PvM & $\begin{array}{l}\text { Lower repair cost } \\
\text { Less equipment } \\
\text { malfunction and } \\
\text { unplanned downtime }\end{array}$ & $\begin{array}{l}\text { Need for inventory } \\
\text { Increased planned } \\
\text { downtime } \\
\text { Maintenance on } \\
\text { seemingly perfect } \\
\text { equipment }\end{array}$ & $\begin{array}{l}\text { Have a likelihood of } \\
\text { failure that increases } \\
\text { with time or use }\end{array}$ & $\begin{array}{l}\text { Have random failures } \\
\text { that are unrelated to } \\
\text { maintenance }\end{array}$ \\
\hline PdM & $\begin{array}{l}\text { A holistic view of } \\
\text { equipment health } \\
\text { Improved analytics } \\
\text { options } \\
\text { Avoid running to failure } \\
\text { Avoid replacing a } \\
\text { component with useful } \\
\text { life }\end{array}$ & $\begin{array}{l}\text { Increased upfront } \\
\text { infrastructure cost and } \\
\text { setup (e.g., sensors) } \\
\text { More complex system }\end{array}$ & $\begin{array}{l}\text { Have failure modes } \\
\text { that can be cost- } \\
\text { effectively predicted } \\
\text { with regular } \\
\text { monitoring }\end{array}$ & $\begin{array}{l}\text { Do not have a failure } \\
\text { mode that can be cost- } \\
\text { effectively predicted }\end{array}$ \\
\hline
\end{tabular}

\section{Predictive Maintenance}

Digitization in enterprises has facilitated to log enormous amount of data about their system status, which was not possible ever before. The logged data opened a new vision for companies and industries to analyse and understand the unknown correlations of the information of the past. Visualizing the past results more accurate predictions for future is possible. Therefore, the more experienced industries in data processing have target to collect the necessary data in order to have higher accuracy predictions. In terms of maintenance, the main goal of predictions is about failures and breakdowns. Predicting failures, not only improve system reliability but also it helps the enterprises in asset management and cost estimations.

\subsection{Environmental Aspects}

United Nations (UN) set 17 goals for sustainable development to be achieved by 2030 . These goals are all interconnected and some of these goals are related to climate change and environmental degradation [11], which is one of the challenges of our time.

From the environmental aspects also, predictive maintenance diminishes carbon footprint in different ways. Firstly, by using maximum life span of a component it helps on material saving. Secondly, by cutting unnecessary maintenance activities less machineries and energy will be used which with a holistic view it has a considerable emission reduction impact. Finally, the time gap before failures gives the opportunity to plan beforehand and increase intervention efficiency. There are many studies that giving special attention to the sustainability aspects of PdM and condition-based maintenance (CBM). Some researches focused on sustainable manufacturing and production frameworks [12]-[16] and some used PdM to lower the emission directly [17], [18].

\subsection{System Reliability}

The reliability-centered maintenance (RCM), was firstly introduced by aviation industry based on a report for preparing maintenance programs of aircrafts [19]. In this report it was mentioned that some failures could not effectively reduce or prevented with scheduled maintenance programs [20]. Considering $\mathrm{DH}$ as a relatively new infrastructure, it takes the attentions to reliability issues. These issues could be divided into security of supply and resilience in disasters, which the latter is out of scope of this paper. There is also an economic 
threat for the utility. This also leads to an interest for reliability of the system. Furthermore, resilient infrastructure is a benefit for the company since they earn money with assets that last longer than the return of investment point.

In terms of reliability, PdM tools and technologies are the most advanced techniques to improve system reliability [2]. PdM has no limitation on number of features that need to be considered in evaluations and it is capable of handle unknown number of variables which deterministic approaches are not able to do so. The newest approaches of PdM also consider the real-time conditions of the assets, which is not only in use for maintenance activities, but also beneficial for optimization of the operation.

\subsection{Asset Management}

According to EN-253:2019 the DH pipes should have a property to be in service for at least 30 years, and based on this time frame, a minimum requirement has been set for the pipe manufacturers that need to be satisfied. But in practice, the pre-insulated bonded pipes last longer than expected. An expert interview conducted by AGFW (German District Heating Association) explains the service life of these pipes between 35 to 70 years (Fig. 1, first column) [21].

\section{Expected Technical Service-Life}

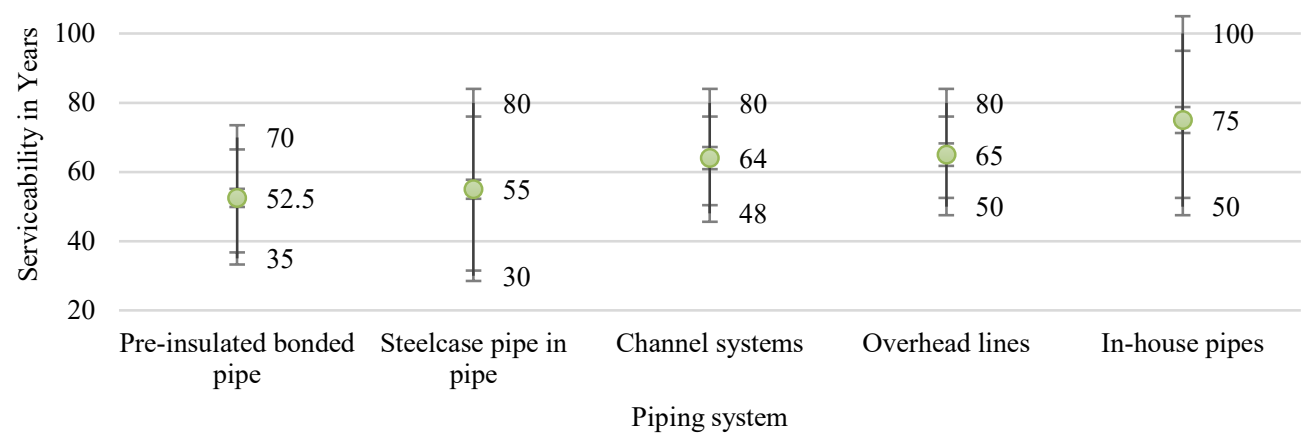

Fig. 1. Expected technical service life of piping systems according to AGFW, FW114 [21].

This dispersion not only makes the maintenance difficult, but also the true value of the asset remains ambiguous, and makes it hard for further planning and developments. Furthermore, $\mathrm{DH}$ is a robust infrastructure and thus there is not so much failure data, and small scale DH networks were built in the recent decades and asset management is not common for those small networks. Even though economic risks may be even higher here. Hence, employing PdM brings another added value to the system and enhance asset management with more precise service life predictions.

\section{Diagnosis and Prognosis Approaches in PdM}

The main task of PdM is estimations of the degradation state of an item. Conventional estimation models for DH pipes are not reliable regardless the evaluation approaches whether 
deterministic or statistic. In parallel, the fourth industry revolution is facilitating and supporting the machineries and components of a system via connecting them with the cyber space. This interconnection opens a new perspective to understand the behaviour of complex systems, and therefore, new means and ideas of diagnosis and prognosis to the complex problems fulfil with highly sophisticated algorithms. Before jumping to the PdM diagnosis and prognosis methods, it is necessary to understand DH system components and the system characteristics for PdM.

\subsection{DH System Characteristics}

An important key factor for a proper maintenance is to understand the ageing and degradation process. Therefore, a system component analysis is required at the beginning stage.

The idea of DH system is to deliver the heat from the heat source(s) to the consumers, and a very simple model of every DH system comprises of minimum three components as follow (Fig. 2):

1. Heat source;

2. Transmission and distribution line;

3. Consumer.

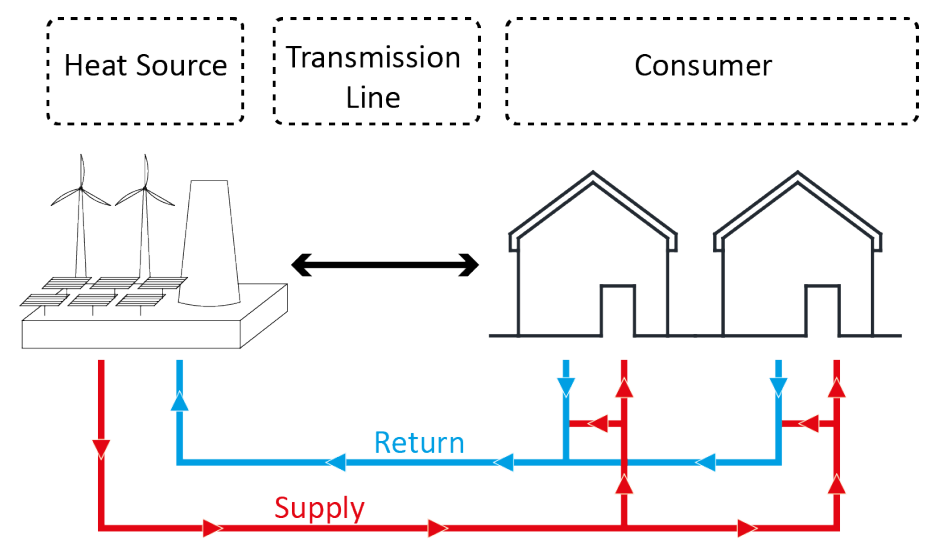

Fig. 2. A scheme of a typical District Heating System.

This paper is focusing on maintenance of distribution network and the suitable PdM methods based on system requirements. Hereupon, the topic will be discussed over the "distribution network".

DH industry is in transition from the $3^{\text {rd }}$ to the $4^{\text {th }}$ generation [22]. In the $3^{\text {rd }}$ generation of DH system, mostly the implemented pipes employing the steel as the medium pipe, rigid polyurethane (PUR) as insulation layer and polyethylene (PE) jacket for protection [23]. Since 1970s, DH industry is employing pre-insulated bonded pipes and need for maintenance stimulated studies concerning ageing of each components of the DH pipes. Investigations in different research projects in different levels as well as national and European revealed the main causes of the ageing in DH pipes. As the pre-insulated bonded pipes are compound materials, the ageing behaviour is interdependent. There are different agents for DH pipe ageing but damage accumulation, thermo-oxidative degradation/gas diffusion and oxidation are respectively major ageing actors for steel pipe, PUR and PE jacket [24]. Table 2 indicates the ageing of DH pipe components and the related ageing models. 
TABLE 2. DH Pipe COMPONENTS, DEgRAdATION TyPe AND SugGeSTED AgEING Model

\begin{tabular}{llll}
\hline Component & Degradation type & Ageing model & Shape \\
\hline Steel pipe & $\begin{array}{l}\text { Damage accumulation } \\
\text { Low-cycle fatigue }\end{array}$ & $\begin{array}{l}\text { Palmgren-Miner rule } \\
\text { Morrow's rule }\end{array}$ \\
\hline Polyurethane Foam & $\begin{array}{l}\text { Thermo-oxidative } \\
\text { Gas diffusion }\end{array}$ & $\begin{array}{l}\text { Arrhenius equation } \\
\text { Fics law }\end{array}$ & \\
\hline Polyethylene Jacket & Oxidation & Arrhenius & \\
\hline Pipe assembly & Complex & $\begin{array}{l}\text { Combined model } \\
\text { required }\end{array}$ & \\
\hline
\end{tabular}

In DH system hydraulic and thermal are the two domains that require control [25]. These two apply different stresses to the network; pressure from the hydraulic and thermal expansion from the temperature variations. Hydraulic and system pressure plays a major role on heat delivery, and in fatigue analysis the stresses applied by system pressure are very small comparing the temperature changes and often neglected [26]. Nevertheless, thermo-oxidation majorly rules the degradation process in PUR and therefore, hydraulic should be considered in predictions.

Weidlich and Schuchardt discussed two fundamental approaches for ageing models of DH pipes and suggested a new combined model is required to integrate the approaches for a higher accuracy [27]. These approaches are:

1. Bottom-up models based on changes in material properties;

2. Top-down models based on statistics and distribution functions.

According to [8], where the degradation process is unobservable, two general types of analysis are most widely used: times series analysis and conditional probability constructions. This is the situation where machine learning takes the lead with the possibility of controlling vast, high dimensional input data.

\subsection{Machine Learning}

Machine Learning (ML) brings together computer science and statistics to enable computers to learn how to do a given task without programming it. The different theory and forms of ML is well explained in [28]-[30]. Understanding the system is decisive to have a decent approach in ML. ML is mainly divided into three groups: Supervised, Semi-supervised and Unsupervised. Deciding to which approach is better for our system depends on our training data. If the data is labelled, supervised learning provide predictions that are more accurate. If the data is not labelled then unsupervised learning is the proper approach. And if the data is partially labelled then semi-supervised learning approach could be employed [31]. When the approach is defined then based on the data type whether it is discrete or continuous, classification or regression algorithms could be selected for the learning application (Fig. 3). 


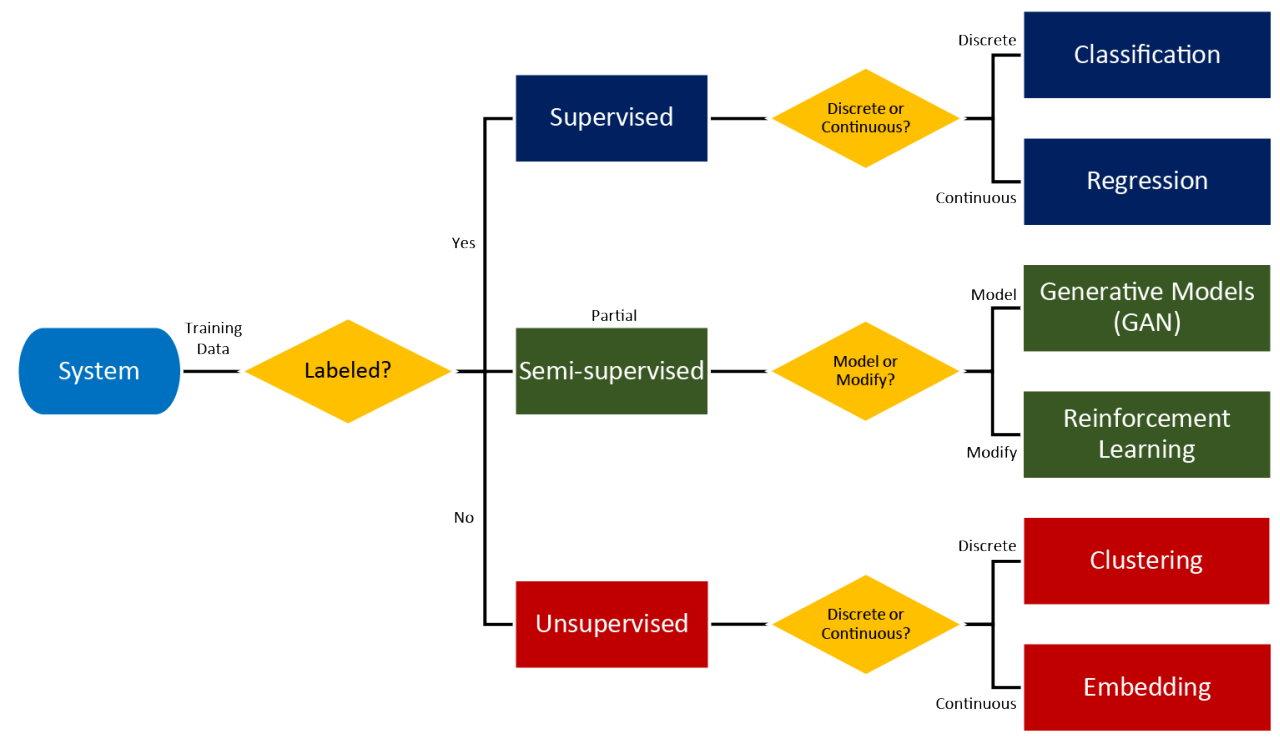

Fig. 3. Types of the Machine Learning based on the input data [32].

In DH distribution line, utilities could exploit their inventory data merge to the damage statistics. This provides a labelled data set which could be exploit for a supervise learning in order to predict the failure on pipes. Of course, the quality of the predictions like all the MLs are highly dependent on the quality of the data and since the DH grids are relatively new infrastructures, high quality data is not available. Therefore, simulations could be a way to compensate the missing data in order to understand the behaviour of ageing in DH pipes.

It is known that the thermo-oxidation in PUR and damage accumulation in steel medium pipe is highly dependent on temperature and temperature changes, thus having the temperature changes of the past is beneficiary for the evaluation of the ageing in the both materials; steel and PUR. Nowadays, utilities predict the heat demand profile to optimize their heat production based on the past data and weather forecast. Based on this principle, it is plausible to use the weather record and the past few years of utility temperature changes for both the supply and return pipes to simulate the temperature changes. The advantage of this simulation is that the available weather data are the true values, and they are not predictions, which possibly results to a higher accuracy. The result of temperature change simulation could be a ground for further learnings and calculations and could be used as input data for cycle counting of fatigue and thermo-oxidative degradation of PUR. With merging these data sets, not only it supports to enrich the sparse data, but also makes it feasible to understand the degradation process and to predict failures.

\section{Artificial Neural Networks}

Without any doubt, 2012 was a great breakthrough for ML and ANN. Deep neural networks significantly outperformed the previous Markov models (HMMs) or Gaussian mixture models (GMMs) [33]. It recognized faces without having to label images [34] and the advent of ImageNet [35], [36]. ANN has shown superior ability in all aspects of ML as well as feature detection, fault classification and prediction with multilayer nonlinear transformations [6]. Since ANN introduced in the late 1940s, different network architectures have been developed 
to address different classification, regression, embedding and clustering problems. The Asimov institute composed a mostly completed chart of all architectures consist of 29 types [37] and thus a study on ANN architecture is required. Meireles et al. conducted a comprehensive review on ANN use in industrial application [38] divided the ANNs in to eleven groups, which the Multilayer Perceptrons (MLPs) were at rank one in usage. According to [32], [39] most frequent ANN architectures are:

1. Multilayer Perceptrons (MLPs);

2. Convolutional Neural Networks $(\mathrm{CNN})$;

3. Recurrent Neural Networks (RNNs).

4. Autoencoders.

The functionality and application of the named architectures has been summarized in Table 3.

TABLE 3. FUNCTIONALITY AND APPLICATION OF ANN ARCHITECTURE IN DIFFERENT INDUSTRIES

\begin{tabular}{|c|c|c|c|}
\hline Architecture & Industry & Application & Functionality \\
\hline \multirow{4}{*}{$\begin{array}{l}\text { Multilayer Perceptrons } \\
\text { (MLPs) }\end{array}$} & Flow rate monitoring & Maintenance & Classification \\
\hline & \multirow{2}{*}{$\begin{array}{l}\text { Thermal performance of } \\
\text { water reactors }\end{array}$} & Fault detection & Clustering \\
\hline & & Control & Regression \\
\hline & Temperature control systems & Optimization & $\ldots$ \\
\hline \multirow{4}{*}{$\begin{array}{l}\text { Convolutional Neural } \\
\text { Networks (CNN) }\end{array}$} & Autonomous mobility & Image processing & \multirow{4}{*}{$\begin{array}{l}\text { Classify, Recognize, and } \\
\text { Gather }\end{array}$} \\
\hline & Face recognition in social & Optimization & \\
\hline & media & Pattern recognition & \\
\hline & Image analysis in health care & Object Detection & \\
\hline \multirow{5}{*}{$\begin{array}{l}\text { Recurrent Neural Networks } \\
\text { (RNNs) }\end{array}$} & Oil and Gas & \multirow{5}{*}{$\begin{array}{l}\text { Predictive } \\
\text { Maintenance } \\
\text { Voice recognition } \\
\text { Extract relevant } \\
\text { insights } \\
\text { Speech to text }\end{array}$} & Prediction \\
\hline & Audio / Temporal signals & & Modelling \\
\hline & Text summarization & & Determine patterns \\
\hline & \multirow[t]{2}{*}{$\begin{array}{l}\text { Conversational Interfaces and } \\
\text { chatbots }\end{array}$} & & \\
\hline & & & \\
\hline \multirow[t]{5}{*}{ Autoencoders } & New characters of animations & \multirow{5}{*}{$\begin{array}{l}\text { Dimensionality } \\
\text { reduction } \\
\text { Data denoising } \\
\text { Feature Extraction } \\
\text { Image Generation }\end{array}$} & Prediction \\
\hline & Fake human images & & Associative Memory \\
\hline & Fix corrupted images & & Sequence to Sequence \\
\hline & Next frame of a video & & prediction \\
\hline & & & \\
\hline
\end{tabular}

\section{Discussion}

In recent years, researches have been used ANN broadly for prediction models and comparing the traditional models with ANN approaches in different disciplines including piping systems. ANN has been used in different piping systems as well as water mains, sewer system, oil, and gas pipelines for condition assessment and predict the grid performance. Al-Barqawi and Zayed combined an analytic hierarchy process (AHP) with ANN to assess condition and prioritize inspection and rehabilitations of the water mains of Canadian municipalities [40], [41]. Senouci et al. developed an ANN base model to predict oil and gas pipeline failures for various failure types as well as mechanical operational and corrosion with an average validity of $92 \%$ [42]. Sharma employed RNN in combination with a long 
short-term memory (LSTM) for predictive maintenance and asset management in oil and gas industry [43].

For DH system based on IoT equipment and data availability of grid, it is more plausible to apply MLP and RNN architecture. In both approaches, the network must be considered as a dynamic system. IoT equipment could provide real-time where available, which is not only useful for monitoring, but also PdM could get benefits of it.

Feature selection plays a decisive role in PdM. For water pipe's degradation, Al-Barqawi and Zayed classified the factors into three main groups as Table 4 . These factors could be used as features in ML where available.

Table 4. Factors Resulting Water Pipe System Deterioration [40]

\begin{tabular}{lll}
\hline Physical factors & Environmental factors & Operational factors \\
\hline Pipe age and material & Pipe bedding & Internal water pressure \\
Pipe wall thickness & Trench backfill & Leakage \\
Pipe vintage & Soil type & Water quality \\
Pipe diameter & Groundwater & Flow velocity \\
Type of joints & Climate & Backflow potential \\
Thrust restraint & Pipe location & Operational and maintenance practices \\
Pipe lining and coating & Disturbances & \\
Dissimilar metals & Stray electrical currents & \\
Pipe installation & Seismic activity & \\
Pipe manufacture & & \\
\hline
\end{tabular}

Although the factors listed in Table 4 are very interesting in model training but mostly this information is not available in DH sector. Furthermore, inspections measures to gather information of the conditions are very limited due to high costs and limited significance of the results.

Treshchenko and Nord listed the most common assumed factors for DH distribution networks, which could be used in system reliability [44]. These factors are as follow:

- Age and installation period

- Soil conditions

- Corrosion

- Previous failures

- Diameter

- Nearby excavation

- Pipe length

- Pressure

- Pipe material

- Dissimilar metals

- Land use

- Seasonal variation

- Temperature levels

- Welding

At the status quo, there is no universal protocol for utilities and asset owners to record this information for DH PdM strategies; hence, approaches might be different from network to network based on the data availability.

\section{Conclusion}

Asset management and security of supply is at importance in DH system as any other infrastructure. Prediction of remaining useful life of the DH pipes are remarkably useful for asset management and maintenance issues. There is no strait forward answer for the ageing 
process of the DH pipes and combination of methods are required. A single ML approach is not adequate to address such a complex system and multiple classification and regression models are required. Based on the size and availability of data, statistical based models and ANN models could be employed. Depend on the DH network characteristics, MLP and RNN architecture seems to be most appropriate architecture for training and predictions. There is no universal protocol for asset owners to record all the necessary data for PdM strategies in $\mathrm{DH}$. Therefore, after creation a successful model it is required to arrange a universal data frame so that the diagnosis model could be applied in all networks with minimum changes. This may also be challenge for utilities and grid operators, not only because of their limitation on data of their own inventory and asset properties, but also implementation of these techniques requires experts, which might not be needed so far in this field.

\section{ACKNOWLEDGEMENT}

The authors acknowledge the financial support by the Federal Ministry for Economic Affairs and Energy of Germany in the project Instandhaltung-FW (project number 03ET1625B).

\section{REFERENCES}

[1] Bevilacqua M., Braglia M. The analytic hierarchy process applied to maintenance strategy selection. Reliability Engineering \& System Safety 2000:70(1):71-83. https://doi.org/10.1016/S0951-8320(00)00047-8

[2] Mobley R. K. An introduction to predictive maintenance. Amsterdam: Elsevier Butterworth-Heinemann, 2002.

[3] Susto G. A., Beghi A., de Luca C. A Predictive Maintenance System for Epitaxy Processes Based on Filtering and Prediction Techniques. IEEE Trans. Semicond. Manufact. 2012:25(4):638-649. https://doi.org/10.1109/TSM.2012.2209131

[4] Holmberg K. E-maintenance. London: Springer, 2010.

[5] Levitt J. Complete guide to predictive and preventive maintenance. New York: Industrial Press, 2011.

[6] Ran Y., et al. A Survey of Predictive Maintenance: Systems, Purposes and Approaches. Ithaca: Cornell University, 2019.

[7] Mobley R. K. Maintenance fundamentals. Amsterdam: Elsevier Butterworth-Heinemann, 2004.

[8] Nachlas J. A. Reliability engineering: Probabilistic models and maintenance methods. Boca Raton: CRC Press, 2017.

[9] Selcuk S. Predictive maintenance, its implementation and latest trends. Proceedings of the Institution of Mechanical Engineers, Part B: Journal of Engineering Manufacture 2017:231(9):1670-1679. https://doi.org/10.1177/0954405415601640

[10] Sharma A. PyTorch on Azure: Deep learning in the oil and gas industry [Online]. [Accessed 23.01.2020]. Available: https://azure.microsoft.com/en-us/blog/pytorch-on-azure-deep-learning-in-the-oil-and-gas-industry/?cdn=disable

[11] UNITED NATIONS. About the Sustainable Development Goals - United Nations Sustainable Development [Online]. [Accessed 19.02.2020]. Available: https:/www.un.org/sustainabledevelopment/sustainable-development-goals

[12] Lindström J., et al. Towards Intelligent and Sustainable Production: Combining and Integrating Online Predictive Maintenance and Continuous Quality Control. Procedia CIRP 2017:63:443-448. https://doi.org/10.1016/j.procir.2017.03.099

[13] Kumar A., Shankar R., Thakur L. S. A big data driven sustainable manufacturing framework for condition-based maintenance prediction. Journal of Computational Science 2018:27:428-439. https://doi.org/10.1016/j.jocs.2017.06.006

[14] Demoly F., Kiritsis D. Asset Optimization and Predictive Maintenance in Discrete Manufacturing Industry. IFAC Proceedings Volumes 2012:45(31):1-6. https://doi.org/10.3182/20121122-2-ES-4026.00033

[15] Golinska P., Fertsch M., Marx-Gómez J. Information Technologies in Environmental Engineering. Berlin, Heidelberg: Springer Berlin Heidelberg, 2011.

[16] Jasiulewicz-Kaczmarek M., Drozyner P. Maintenance Management Initiatives Towards Achieving Sustainable Development. In: Golinska P., Fertsch M., Marx-Gómez J. (eds) Information Technologies in Environmental Engineering. Environmental Science and Engineering, vol. 3. Springer, Berlin, Heidelberg.

[17] Pitstop Connect. Reduce Vehicle Emissions with Predictive Maintenance - Climate CoLab [Online]. [Accessed 5.02.2020]. Available: https:/www.climatecolab.org/contests/2018/helping-small-medium-businesses-go-lowcarbon-ontario/c/proposal/1334500 
[18] United States Environmental Protection Agency. Equipment Upgrades and Preventive Maintenance Improve Performance and Reduce SF6 Emissions. US: EPA, 2017.

[19] Moubray J. Reliability-centred maintenance. Amsterdam: Elsevier Butterworth-Heinemann, 1997.

[20] Nowlan F. S., Heap H. F. Reliability-Centered Maintenance. US: United Airlines, 1978.

[21] AGFW. FW 114 - Instandhaltungsstrategien und Rehabilitationsplanung - Mindestanforderungen. (Maintenance strategies and rehabilitation planning -minimum requirements.) Frankfurt am Main: AGFW, 2013 (in German)

Lund H., et al. $4^{\text {th }}$ Generation District Heating (4GDH): Integrating smart thermal grids into future sustainable energy systems. Energy 2014:68:1-11. https://doi.org/10.1016/j.energy.2014.02.089

[22] Schuchardt G. K., Weidlich I. Sensitivity analysis of the conception of small scale district heating networks on the thermal conductivity of the surrounding soil. Energy Procedia 2017:128:136-143. https://doi.org/10.1016/j.egypro.2017.09.028

[23] Pourbozorgi Langroudi P., Weidlich I. Entwicklung von neuen und verbesserten Instandhaltungsstrategien für kleine und große Wärmeverteilnetze durch Kombination statistischer Alterungsmodelle mit materialbasierten Nutzungsdauermodellen: AP1. (Development of new and improved maintenance strategies for small and large heat distribution networks by combining statistical aging models with material-based service life models: AP1.) unpublished intermediate report, project number 03ET1625B. Berlin: Federal Ministry for Economic Affairs and Energy of Germany, 2019. (in German)

[24] Gambarotta A., et al. A Library for the Simulation of Smart Energy Systems: The Case of the Campus of the University of Parma. Energy Procedia 2017:105:1776-1781. https://doi.org/10.1016/j.egypro.2017.03.514

[25] Christensen R. Fatigue Analysis of District Heating Systems. 1999.

[26] Weidlich I., Schuchardt G. K. New Approach for Asset Management in District Heating (DH) Networks. Energy Procedia 2017:113:22-27. https://doi.org/10.1016/j.egypro.2017.04.007

[27] Chollet F. Deep learning with Python. Shelter Island. NY: Manning, 2018.

[28] Raschka S. Python machine learning: Unlock deeper insights into machine learning with this vital guide to cuttingedge predictive analytics. Birmingham, Mumbai: Packt Publishing open source, 2016.

[29] Murphy K. P. Machine learning: A probabilistic perspective. Cambridge: MIT Press, 2012.

[30] Brunton S. L., Kutz N. J. Data-driven science and engineering: Machine learning, dynamical systems and control. Cambridge: Cambridge University Press, 2019.

[31] Brunton S. Intro to Data Science: Types of Machine Learning 2 [Online]. [Accessed 17.02.2020]. Available: https:// www.youtube.com/watch?v=0_1KUPYEYyY\&list=PLMrJAkhIeNNQV7wi9r7Kut8liLFMWQOXn\&index=10

[32] Hinton G., et al. Deep Neural Networks for Acoustic Modeling in Speech Recognition: The Shared Views of Four Research Groups. IEEE Signal Process. Mag. 2012:29(6):82-97. https://doi.org/10.1109/MSP.2012.2205597

[33] Le V Q., et al. Building high-level features using large scale unsupervised learning. Itacha: Cornell University, 2011.

[34] Krizhevsky A., et al. Classification with Deep Convolutional Neural Networks. Advances in Neural Information Processing Systems 2012:25(2):1097-1105.

[35] Pereira F., et al. Advances in Neural Information Processing Systems 25. Curran Associates, 2012.

[36] van Veen F. The Neural Network Zoo. The Asimov Institute, 2016. [Online]. [Accessed 18.02.2020]. Available: https:// www.asimovinstitute.org/neural-network-zoo

[37] Meireles M.R.G., Almeida P.E.M., Simoes M. G. A comprehensive review for industrial applicability of artificial neural networks. IEEE Trans. Ind. Electron. 2003:50(3):585-601. https://doi.org/10.1109/TIE.2003.812470

[38] Brownlee J. Deep Learning With Python: Develop Deep Learning Models On Theano And TensorFlow Using Keras. Vermont Victoria: Machine Learning Mastery, 2016.

[39] Al-Barqawi H., Zayed T. Condition Rating Model for Underground Infrastructure Sustainable Water Mains. J. Perform. Constr. Facil. 2006:20(2):126-135. https://doi.org/10.1061/(ASCE)0887-3828(2006)20:2(126)

[40] Al-Barqawi H., Zayed T. Infrastructure Management: Integrated AHP/ANN Model to Evaluate Municipal Water Mains' Performance. J. Infrastruct. Syst. 2008:14(4):305-318. https://doi.org/10.1061/(ASCE)10760342(2008)14:4(305)

[41] Senouci A., et al. A model for predicting failure of oil pipelines. Structure and Infrastructure Engineering 2014:10(3):375-387. https://doi.org/10.1080/15732479.2012.756918

[42] Sharma A. PyTorch on Azure: Deep learning in the oil and gas industry [Online]. [Accessed 30.10.2019]. Available: https://azure.microsoft.com/en-us/blog/pytorch-on-azure-deep-learning-in-the-oil-and-gas-industry/?cdn=disable

[43] Tereshchenko T., Nord N. Importance of Increased Knowledge on Reliability of District Heating Pipes. Procedia Engineering 2016:146:415-423. https://doi.org/10.1016/j.proeng.2016.06.423 